\title{
Féeries
}

Études sur le conte merveilleuX, XVII ${ }^{e}$ XIXe siècle

\section{Du conte oriental comme encyclopédie narrative}

Dépôts de savoir et matière de fiction dans les contes de Gueullette

The Oriental Tale as Narrative Encyclopoedia: Layers of Knowledge and Stuff of Fiction in Gueullette's Tales

Jean-François Perrin

\section{OpenEdition Journals}

Édition électronique

URL : http://journals.openedition.org/feeries/714

DOI : 10.4000/feeries.714

ISSN : 1957-7753

Éditeur

UGA Éditions/Université Grenoble Alpes

Édition imprimée

Date de publication : 1 juillet 2009

Pagination : 189-221

ISBN : 978-2-84310-140-3

ISSN : $1766-2842$

Référence électronique

Jean-François Perrin, « Du conte oriental comme encyclopédie narrative », Féeries [En ligne], 6 | 2009, mis en ligne le 15 septembre 2010, consulté le 08 septembre 2020. URL : http:// journals.openedition.org/feeries/714; DOI : https://doi.org/10.4000/feeries.714 


\title{
DU CONTE ORIENTAL COMME ENCYCLOPÉDIE NARRATIVE
}

\author{
DÉPÔTS DE SAVOIR ET MATIÈRE DE FICTION \\ DANS LES CONTES DE GUEULLETTE
}

$L$

'UNIVERS DES CONTES DE GUEULLETTEI procède d'une immense expérience de lecteur. S’il est une œuvre pour justifier à sa façon le fameux aphorisme mallarméen, " lire - cette pratique $-{ }^{2}$ ", c'est bien la sienne en effet. Tout ici atteste la présence virtuelle d'une sorte de fichier documentaire où se trouvent consignés et classés extraits d'œuvres, articles de dictionnaires, citations de différents formats, noms propres, données historiques, géographiques, ethnographiques, scénarios de nouvelles, de contes, objets et décors merveilleux, chapitres de romans à utiliser, séquences discursives en philosophie, en théologie musulmane ou chrétienne, scénarios de nouvelles, de contes, anecdotes puisées dans une foule de relations de voyages, etc. C'est ainsi que le chercheur verra reparaître à différents moments de l'œuvre tel ou tel chapitre de Béroalde de Verville, tels ou tels articles de la Bibliothèque orientale concernant la geste de Baharam, ou tel épisode légendaire du Caherman Nameh, tel ou tel scénario tiré de Straparole ou de La Calprenède, tel ou tel nom propre désignant ici une princesse vertueuse, là un héros malicieux, ou encore

I. Sa production contée court de I7I2 à I732, incluant dans l'ordre Les Soirées Bretonnes (1712); Les Mille et Un Quarts d'Heure, Contes Tartares (1715, augmentés en 1723) ; Les Aventures merveilleuses du mandarin Fum-Hoam, Contes Chinois (1723); Les Sultanes de Guzarate, Contes Mogols (1732). Il publie encore en 1759 une édition complétée par ses soins des Mille et Une Heures, Contes Péruviens, parus anonymement en 1733. Une édition critique de l'ensemble de ces recueils est à paraître aux éditions Champion ("Bibliothèque des Génies et des Fées ", vol. 9), réalisée par une équipe composée de Christelle Bahier-Porte pour les Soirées Bretonnes; Marie-Françoise Bosquet et Régine Daoulas pour les Contes Péruviens ; Carmen Ramirez pour les Contes Tartares; JeanFrançois Perrin pour les Contes Chinois et les Contes Mogols.

2. Mallarmé, Le Mystère dans les Lettres. CEuvres complètes, Paris, Gallimard, "Pléiade ", 1945, p. 386-387. 
telle mystification montée par un sultan dérobant sa vue pour mieux régner, conformément à un imaginaire du despote oriental qu'ont accrédité les relations des voyageurs ${ }^{3}$. Qu'il s'agisse de la confection de ses récits cadres, de l'assemblage de ses contes en recueils, de ses fameuses notes documentaires, Gueullette joue en virtuose d'un répertoire de fiches qu'il bat et rebat, consulte et insère dans la mise en œuvre de ses recueils. Ce répertoire, il le nomme dans son testament ses "Miscellanées " où sont consignées en extraits l'intégralité des lectures de toute sa vie ; peut-être sont-elles à jamais perdues ${ }^{4}$, mais on peut sans doute se faire une idée de leur organisation en feuilletant ce qu'on a conservé de ses archives historiques touchant la jurisprudence criminelles, dont la précision documentaire et les principes de classement visiblement destinés à une consultation efficace et rapide, permettent assez aisément de concevoir que ce polygraphe éclairé ne lisait jamais sans avoir en vue la préparation d'une tâche ou d'un ouvrage : histoire du théâtre, archives criminelles, travail d'édition, recueil de contes.

Cette pratique de lecture orientée vers l'écriture dépasse par ses enjeux et ses résultats ce qu'étaient probablement ses intentions conscientes, de par la diversité et l'ampleur des secteurs de la bibliothèque qui sont ainsi mis en relation synchronique, toutes époques et tous genres mêlés. Gueullette connaît bien le conte de fées du XvII ${ }^{\mathrm{e}}$ siècle - et extrêmement bien le conte oriental moderne depuis Galland : Sultanes de Perse, Mille et Un Jours, Aventures d'Abdalla; il est aussi l'éditeur de la traduction Galland des Fables de Bidpä̈, autrement dit le Pantchatantra indien ou le Kalila et Dimna arabe, dont des dizaines de versions circulent en Orient et en Europe depuis des siècles ${ }^{6}$. Il a édité Le Petit Jehan de Saintré et l'Histoire de très noble et chevalereux Gérard comte de Nevers, dont les préfaces et l'annotation montrent qu'il lit le vieux français et qu'il a une connaissance

3. "Omnivoyant mais invisible, le despote tend toujours à se constituer comme un pur être de regard, à la fois périphérique et central, enveloppant et enveloppé, puisqu'il est ce regard qui, dans tous les autres est supposé vous regarder, et ce regard unique qui, du centre du Palais, surplombe la Ville, l'Empire, le Monde. " (Alain Grosrichard, Structure du sérail, Seuil, 1979, p. 75-77)

4. Dans son ouvrage Un magistrat du XVIII siècle, ami des Lettres, du théatre et des plaisirs, ThomasSimon Gueullette, Paris, Droz, 1938, J.-E. Gueullette écrivait ne posséder qu'un volume de ces Miscellanées.

5. Archives T. S. Gueullette. Cartons cotés AD/III/I à 40. Centre historique des Archives nationales, Hôtel de Soubise. Carton AD/III/I.B. "Arrêts et jugements criminels » (II9I-I59I). Pour le point de vue de l'historien, voir Robert Anchel, Crimes et châtiments au XVIII e siècle, Paris, Perrin, I933.

6. Voir l'introduction d'André Miquel à sa traduction d'Ibn al-Muqaffac, Le Livre de Kalila et Dimna, Klincksieck, I980. 
sérieuse des romans médiévaux et même de la littérature historique et critique disponible à leur propos. On ne s'étonnera pas dès lors que l'étude des sources de ses Contes Tartares fasse rencontrer toute une hypotextualité médiévale : exempla, farces, soties, etc. que du reste connaissait fort bien la philologie érudite du $\mathrm{XIX}^{\mathrm{e}}$ siècle, l'érudition orientaliste comprise, comme le montrent, entre autres exemples, les références à Gueullette chez Loiseleur-Deslongchamps ${ }^{7}$, dans les études comparatistes anglo-saxonne ${ }^{8}$ ou encore dans l'immense somme de Victor Chauvin sur la diffusion européenne des Mille et Une Nuits'. Le Gueullette des Contes Tartares figure en effet dans les annotations des Fabliaux édités par Legrand d'Aussy ou dans celles des Facétieuses nuits ${ }^{10}$ ou des recueils de Bandello, comme un des chaînons familiers de la transmission de toute cette tradition narrative et morale. Quand on connaît l'envergure de la diffusion européenne de son œuvre conté au XVIII ${ }^{\mathrm{e}}$ siècle et au-delà ${ }^{\mathrm{II}}$, on prend la mesure de ce qui se joue certainement à travers lui d'une vaste translatio en cours de l'ancienne littérature vers le siècle des Modernes.

7. Auguste-L.-A. Loiseleur-Deslongchamps, Essai historique sur les contes orientaux et sur les Mille et Une Nuits. Introduction à son édition des Mille et Une Nuits, Paris, I838.

8. John Dunlop, The History of Fiction, 3 vol., Édimbourg, I8I6. W. A. Clouston, Popular Tales and Fictions: Their Migrations and Transformations, 2 vol., Édimbourg et Londres, I887. Rééd. Kessinger Publishing, 2006.

9. V. Chauvin, Bibliographie des ouvrages arabes, ou relatifs aux Arabes publiés dans l'Europe chrétienne de I81o à 1884, I2 vol. (les vol. IV-VII [1900-1908] concernent les Mille et Une Nuits et leur diffusion), Liège-Leipzig, I892-I922.

Io. Il y a bien un " moment " Gueullette de cette chaîne de transmission, et ce moment en relaie un autre, celui de la diffusion européenne et française du recueil de Straparole, qui a contribué à donner au conte de fées "à la française " une sorte d'unité formelle. (Voir Ruth B. Bottigheimer, "France's First Fairy tales: The Restoration and Rise Narratives of Les facetieuses nuictz du Seigneur Francois Straparole ", Marvels \& Tales: Journal of Fairy-Tale Studies, vol. 19, n I, 2005, p. 17-31). Les contemporains de Gueullette ont bien vu qu'une bonne part des matrices narratives des Contes Tartares était empruntée à Straparole (mais il pille aussi Bandello et Boccace). C. Ramirez présente par exemple, dans notre édition critique, la notice élogieuse du marquis de Voyer de Paulmy d'Argenson (Mélanges tirés d'une grande bibliothèque) concernant les réécritures de Straparole dans les Contes Tartares.

II. Si ses Soirées Bretonnes n'eurent guère de succès, les trois recueils suivants ont connu une fortune européenne remarquable jusqu'au XIX ${ }^{\mathrm{e}}$ siècle : onze éditions françaises des Contes Tartares publiées au XVIII ${ }^{e}$ siècle avant celle du Cabinet des fées, et une vingtaine d'éditions dans les autres langues européennes. Au XIX ${ }^{\mathrm{e}}$ siècle, on les réédite encore en Angleterre, en Allemagne, en Espagne et au Portugal. Le XviII ${ }^{\mathrm{e}}$ siècle voit paraître trois éditions françaises des Contes Chinois avant le Cabinet des fées, et leur traduction est plusieurs fois rééditée en Angleterre et en Allemagne (encore cinq éditions anglaises au XIx ${ }^{e}$ siècle). Concernant les Sultanes de Guzarate, ce sont six éditions françaises au XviII ${ }^{\mathrm{e}}$ siècle avant celle du Cabinet des Fées, et sur le plan des traductions, cinq éditions anglaises, deux allemandes, une italienne et une russe. Au début du XIx ${ }^{\mathrm{e}}$ siècle, l'ensemble des contes orientaux de Gueullette seront réédités en anglais dans l'anthologie de H. Weber : Tales of The East (1812). 
Mais s'il y a donc bien un " moment " Gueullette de cette chaîne de transmisssion, cette connexion ou conjonction des univers culturels orientaux et européens opérée dans ses recueils de contes orientaux n'est sans doute elle-même que l'une des facettes du moment proprement moderne de l'émergence ou de la réémergence d'un partage fort ancien. Huet ${ }^{\mathrm{I}}$, d'Herbelot, Galland l'observent et le disent : il y a de puissantes parentés entre certaines figures et certains récits des deux traditions culturelles et littéraires. Galland cite l'Histoire de Pierre de Provence et de la belle Maguelonne dans une note à l'Histoire de Camaralzaman, d'Herbelot rapproche la ginne Mergian-Banou de la grande fée " déconnuë » des Amadis $^{13}$. C'est certainement en toute conscience de ces parentés, voire de cette commune origine à laquelle croyait le monde savant de son époque ${ }^{\mathrm{I}}$, que Gueullette a travaillé dans ses recueils orientaux à hybrider, si l'on peut dire, l'ancienne matière narrative européenne dans le goût du conte oriental déclenché par le succès de Mille et Une Nuits et des Mille et Un Jours. Dans cette entreprise, rien n'aurait été possible sans le monumental ouvrage de Barthelemi d'Herbelot, la Bibliothèque orientale, dont Galland avait écrit dans la préface qu'il lui avait donnée, qu'outre son caractère de somme encyclopédique, elle recèle " tant d'autres livres de fables morales, de collections de proverbes, de sentences ou de maximes, de paroles remarquables et de bons mots, de contes divertissants et d'histoires fabuleuses que nous appelons romans ". Des Mille et Un Quarts d'Heure aux Sultanes de Guzarate, Gueullette s'est servi presque à chaque page de la Bibliothèque orientale. Il y a pris les noms de l'essentiel de son personnel romanesque, avec leur traduction ; il y a trouvé la base du conte cadre des Aventures du mandarin Fum-Hoam et une partie de celle des Mille et Un Quarts d'Heure; il y a puisé une bonne cinquantaine de scénarios, il en a tiré l'essentiel de son annotation documentaire, le plus souvent littéralement recopiée ; il s'en est servi pour construire en centon l'apologie de la religion musulmane qui conclut les Contes Chinois; il y a recopié des vers de Saadi, des sentences et des proverbes, des anecdotes

I2. Dans sa Lettre-Traité sur l'Origine des romans (1670).

13. Bibliothèque orientale (1697), art. "Mergian".

I4. «Ils [les contes] sont assez anciens écrit Galland à Gisbert Cuper, et la communication qu'il y a eu de ces pays avec le Levant du temps des croisades fait que les auteurs de Vieux romans en ont tiré beaucoup de choses. " Lettre du 3 octobre 1704, citée dans M. Abdel-Halim, Antoine Galland, sa vie et son cuvre, Nizet, 1964, p. 299 ; même écho au Journal des savants rendant compte des deux premiers tomes des Nuits: "La conformité qui se trouve entre quelques-uns de ces contes et ceux que nous lisons dans les ouvrages de nos auteurs européens, nous porterait à croire que ce livre arabe ne leur a pas été entièrement inconnu. » (Abdel-Halim, ibid.) 
drolatiques, des canevas de farces, qu'il a ensuite greffés en ornementation pour ses contes, en contrepoint avec la veine féerique et ses objets magiques : main enchantée, herbe lionnée, statues de vérité, dragons et bêtes de songes, etc. Il y a trouvé les climats épico-légendaires issus des gestes des Solimans, des Caïanides, du Houschenk nameh, du Caioumarath nameh, du Shâh nameh, ou encore du Coran. Les contes de Gueullette, d'une certaine façon, constituent ainsi une sorte d'abrégé " grand public » de la Bibliothèque orientale, un abrégé très fidèle à sa source que le conteur plagie souvent au mot près et dont, sur le plan narratif, il transmet les noyaux durs, se chargeant de les agencer, de les apparier, de les amplifier et de les ornementer, en utilisant pour cela d'autres sources, comme nous verrons. Il y aurait ainsi tout un travail à faire sur la façon dont le conte oriental du XVIII ${ }^{\mathrm{e}}$ siècle, par Galland, par Pétis, par Gueullette, transmet effectivement jusqu'au XIX ${ }^{e}$ siècle à travers le Cabinet des fées, une certaine idée para «savante » de l'Orient, un Orient raconté au plus près de ce qui, de sa source, est censé avoir irrigué les vieux récits d'Occident.

Un autre corpus de la prose narrativo-documentaire du XVII siècle qui trame les recueils de Gueullette presque au même niveau que la Bibliothèque orientale, est constitué du tissu des relations de voyages - cela des plus fameux : Chardin, Tavernier, Thévenot, Bernier, le Bruyn -, aux minores (Le Blanc, Olearius) ; avec aussi les Lettres édifiantes, les relations de missions, et les traités apparentés. Cette littérature, il la connaît par cœur. Il épluche Chardin avec le même soin et les mêmes objectifs littéraires qu'avec d'Herbelot. Il y puise anecdotes, maximes, sentences et vers ; il y recopie des passages entiers touchant les mœurs, la politique, la religion, il y découpe des " choses vues", qu'il greffe dans ses contes avec le reste. Il en a une connaissance véritablement intime. Mais s'il pille consciencieusement les voyageurs d'Orient, il utilise aussi les ouvrages de Lafitau, La Potherie, LeClerq sur les Indiens d'Amérique du Nord, puisant ici un mythe complet transposé en conte chinois, et là des éléments documentaires transposés, matière de rêve oblige, dans les mœurs d'une peuplade anthropophage de la mer d'Oman ${ }^{15}$. Il y a une curiosité ethnographique insistante dans les recueils de Gueullette, amenant sous couvert d'exotisme, certains aspects originaux de l'observation conduite par les missionnaires sur les mœurs des peuples avec lesquels ils ont vécu. Il serait ainsi possible de dégager chez lui, par exemple, toute une problématique comparatiste concernant les mœurs sexuelles : coutumes et rituels de mariage,

I5. Aventures [...] du mandarin Fum-Hoam, XxIv soirée ; Sultanes de Guzarate, XxIv soirée. J’y reviens plus loin. 
rapports de filiation, interdit de l'inceste, monogamie et polygamie, etc. La littérature de voyage (celle de l'époque non sans quelques excursions chez Marco Polo ou Christophe Colomb) nourrit ainsi, à côté de la Bibliothèque orientale et des dictionnaires géographiques de Baudrand et de Noblot, toute la documentation des notes des recueils et une bonne part de la matière anecdotique (évidemment transposée). On ne forcerait ainsi qu'à peine la vérité en disant que les recueils de Gueullette, pris dans leur ensemble, peuvent être lus comme une micro-bibliothèque des "sciences humaines " de son temps, systématiquement traitées sous l'angle de leur potentiel narratif et fictionnel.

On tentera d'en donner une idée dans les analyses qui vont suivre, en s'appuyant de façon privilégiée sur deux recueils qui se signalent dans cette œuvre par leur couplage explicite, puisque leurs récits cadres relient par une filiation de parenté les deux figures de sagesse qui y mènent le jeu, et que l'on peut y dégager la cohérence et l'approfondissement d'une réflexion en acte sur le rapport des discours de croyance avec les mécanismes de la fiction narrative. Il s'agira des recueils intitulés Les Aventures merveilleuses du mandarin Fum-Hoam, Contes Chinois (1723) et Les Sultanes de Guzarate ou les songes des hommes éveillés, Contes Mogols (1732). On développera d'abord une analyse des composantes "didactiques " ironiquement affichées du recueil de contes orientaux selon Gueullette : les Contes Chinois en fourniront la matière ; on poursuivra par une étude des rapports entre croyance et surdétermination fictionnelle des discours de savoir, à partir cette fois des Contes Mogols.

\section{Didactique de la fiction conteuse : un recadrage sceptique}

Dans le recueil des Contes Chinois, on s'intéressera d'abord au travail proprement didactique, explicite et implicite, de Gueullette, dans son utilisation de sa documentation à l'usage de l'information des lecteurs. On développera ensuite une étude de la dimension critique de la poétique du récit cadre envisagé comme dispositif de relecture sceptique des systèmes de croyance présidant aux hypotextes documentaires dont il est inspiré. On poursuivra en travaillant, à partir de quelques thèmes symptomatiques, les enjeux de la reprise dans les séquences contées elles-mêmes, d'une matière issue ou plagiée de la documentation orientale ou des récits de voyage.

Les notes documentaires en bas de page, qui prennent une extension croissante au fil des recueils, ont été jusqu'ici le seul aspect sérieusement 
étudié de la poétique de Gueullette ${ }^{16}$. Lui-même a d'ailleurs insisté dans l'avant dire des Sultanes de Guzarate, sur le soin qu'il a pris de cette dimension didactique très affichée qui caractérise sa manière ${ }^{17}$. Il s'inscrit néanmoins là dans une claire filiation à la pratique des deux savants qui ont lancé le genre du conte oriental en France : soit travailler, dans les notes ou dans des commentaires informatifs insérés au récit même, à informer la lectrice ou le lecteur des réalités géographiques et historiques, sociales et culturelles des pays évoqués ${ }^{18}$. À cet égard, on doit relever la maîtrise qu’il révèle de sa matière documentaire : outre ce qui provient d'une consultation "alphabétique " des dictionnaires ou des index, tout un pan de l'annotation relève en effet souvent d'une autre méthode de préparation, comme en témoignent quelques exemples tirés des Contes Chinois. Ainsi de certains noms propres traduits en note de l'arabe ou du persan : si l'on veut trouver où il a puisé dans d'Herbelot la note : "Gundogdi en persien signifie aurore ou jour naissant ${ }^{19}$ ", c'est à l'article " Dun Dogdi » qu'il faut aller le chercher. Autre exemple de cas où l'approche alphabétique défaille : le nom de " ce prince appelé Dilsenghin, c'est-à-dire cœur de pierre », adversaire du roi de Géorgie dans le récit cadre ${ }^{20}$, que Gueullette est allé puiser dans l'article "Asfendia'r ": "Sam fils de Zal était surnommé Dil-Senghin, cœur de pierre, et on le met au nombre des plus vaillants capitaines dont les anciennes histoires de Perse fassent mention. »

I6. Dans l'ensemble, ces études s'accordent sur le caractère distancié, voire ironique, de la pratique de la note didactique ou savante chez Gueullette. R. Jomand-Baudry : "Signification des notes auctoriales dans la fiction orientale du début des années 1730 ", dans Le Roman des années trente, la génération de Prévost et de Marivaux, (éd. A. Rivara et A. McKenna), P. U. St-Étienne, 1998, p. I3I-I43 (les dernières pages sont consacrées aux Sultanes). C. Bahier-Porte, "Les notes dans les premiers contes orientaux ", Féeries, n 2, Grenoble, Ellug, 2004-205, p. 9I-I08. C. Langle, "Questions et hypothèses sur l'usage des sources dans Les Sultanes de Guzarate ", Féeries, $\mathrm{n}^{\circ}$ 2, ibid., p. 6I-90.

17. " Il me reste à dire que ce n'est pas sans raison que j'ai mis à ces aventures, des notes géographiques et historiques un peu plus amples que l'on n'a coutume de le faire en pareil cas ; outre qu'il y a nécessairement dans ces sortes d'ouvrages des endroits qui ont besoin d'explication, surtout pour les dames, j'ai cru devoir les appuyer, et principalement ceux qui regardent l'Histoire fabuleuse, de l'autorité de la Bibliothèque orientale, ou des plus célèbres voyageurs qui ont parcouru ces vastes pays, persuadé que le lecteur ne me saura pas mauvais gré de ces remarques, qu'il sentira bien m'avoir coûté beaucoup de temps et de lecture. " (Contes Mogols, "Avis au lecteur ", dernier $\$($ sp.), Paris, Libraires associés, I765; j'utilise ici cette édition dans l'ensemble fidèle à l'originale, malgré le titre général adopté : Les Mille et Une Soirées, qui n’est pas de Gueullette)

18. Pour le travail de Galland sur ce plan, voir Sylvette Larzul : Les traductions françaises des Mille et Une Nuits, Paris, L'Harmattan, 1996.

19. "Histoire de Malekalsalem, roi de Géorgie ", dans Les Avantures merveilleuses du mandarin Fum-Hoam, Contes Chinois, t. I, Paris, J.-B. Mazuel, I723, p. Io.

20. Ibid., p. II. 
Ainsi, comme on l'a suggéré plus haut à propos de sa matière narrative, Gueullette semble disposer d'un répertoire complexe de données de tous ordres, recopiées de ses lectures et classées dans la perspective d'une utilisation littéraire ; le cas du nom de Dilsenghin n'est en effet pas isolé : l'essentiel de ses emprunts, non seulement n'est pas signalé (ce qu'il ne s'agit évidemment pas de lui reprocher), mais surtout ne peut se retrouver par la seule consultation alphabétique des entrées de dictionnaires. Seule une lecture assez complète de celui de d'Herbelot par exemple, permet de découvrir les passages (parfois très conséquents, parfois très brefs) sélectionnés pour être montés dans le texte. C'est notamment le cas dans le sermon de Mohammed Mehdi au début de la XLVI ${ }^{\mathrm{e}}$ soirée : tout le paragraphe est un (re)montage d'une séquence de l'article «Dunia » chez d'Herbelot, par exemple :

Quand on est bien persuadé de cette vérité, peut-on s'attacher au monde ? Ô homme! Si tu t'y arrêtes, tu connaîtras que ce n'est que le fantôme d'un songe, et puisqu'il n'est capable de te fournir que douleur et qu'affliction, pourquoi marques-tu tant d'empressement pour des biens périssables, pendant que tu en abandonnes d'éternels ? Que ton sort est déplorable quand tu t'écartes de la voie de la justice et de la loi du saint Prophète! Ne dresse point sur la terre, (nous dit-il) des tentes qui y soient attachées avec des piquets, et ne te charge point inutilement d'un bagage qu'il faut toujours tenir emballé, et tout prêt à partiir ${ }^{21}$.

Mais Gueullette peut aussi bien réutiliser un distique de Saadi prélevé dans une page de Chardin : "Ô grand prophète ami de Dieu, l'éloge de ta gloire est parfaite dans le verset Toulak, et celui de ta bonté dans le Faha et Jesim ${ }^{22}$. " On n'ira pas plus avant ici dans le domaine de l'annotation en bas de page, sinon pour poser un jalon vers une lecture ironiste de son côté systématique, qu’on retrouvera avec le recueil suivant.

2I. Ibid., t. II, p. 27I. Voici la source : "Hussain Vaêz dans sa paraphrase sur le chapitre Nessa de l'Alcoran, où il est dit $[\ldots]$ : la vie de ce monde n'est que vanité $\&$ tromperie, cite ces vers persans du Methnevi : "Si vous vous y arrêtez, il parle du monde, vous connaîtrez que ce n'est que le fantôme d'un songe. [...] Nezami autre poète persan s'écrie : Que celui-là est heureux qui pense, \& qui après y avoir bien pensé se délivre pour toujours des embarras du monde! [...] Ne dressez point en ce monde de tentes qui soient attachées avec des piquets : Et ne vous chargez point inutilement d'un bagage quil faut toujours tenir emballé pour partir. [...] Le même Hussain Vaêz cite sur le chapitre de Houd ces vers persans: C'est une grande folie que de donner le capital \& le fond de sa religion pour les biens de ce monde [...]/ Vous recevez des biens périssables, \& vous en abandonnez d'éternels./ La prudence ne vous fait-elle pas voir clairement que ce marché n'est pas légitime?" " (B.O., article «Dunia », je souligne.)

22. Contes Chinois, XLv soirée, ouvr. cité, t. II, p. 263 ; vers cités par Chardin au t. V de ses Voyages [...] en Perse et autres lieux de l'Orient, Amsterdam, I7II, p. 273. 
Cette dimension ironiste ou critique, on doit avant tout la faire apparaître dans la structure qui conditionne toute la réception du recueil, et qui est le dispositif de son encadrement narratif, corrélé avec le thème de la métempsycose. À l'époque où Gueullette écrit, le thème de la transmigration des âmes est dans l'air du temps, je l'ai montré naguère ${ }^{23}$. Il est très présent dans la littérature de voyage, en philosophie, chez Bayle, chez Locke (Essai II, 27), Leibniz, les platoniciens de Cambridge, dans les dictionnaires et l'Encyclopédie, etc. Ce thème passionne sans doute parce qu'il engage en termes franchement dualistes le problème des rapports du corps et de l'âme ; et dans les situations de transmigration volontaire que décrivent surtout ses variations littéraires, la collaboration des deux substances tourne à l'aporie lorsqu'un corps est usurpé par une âme étrangère (voire par plusieurs). Une situation si problématique est en général le ressort de l'intrigue ${ }^{24}$; mais sur le plan du discours, l'éternelle question du dualisme et du monisme peut aussi s'y trouver thématisée dans un dialogue : c'est le cas dans l'épisode des Soirées Bretonnes où Gueullette paraphrase ce qu'avait fait sur ce plan Béroalde de Verville ${ }^{25}$. Le prince Entendement vient de lire avec scepticisme le "Traité de la métempsycose » de Pythagore : le vieux sage apparaissant alors, le prince demande comment une âme peut s'unir à un autre corps que le sien; Pythagore répond que l'âme est immatérielle, donc atopique : la matière ne lui est qu'organe ; mais il faut une preuve tangible : le sage anime une tourterelle, puis revenu à sa forme précédente, il montre au prince qu'il a conservé tout son jugement durant la transmigration, puisqu'il a observé au plafond un lambris étrangement percé ; le prince y trouvera certaines lamelles précieuses et le conte se poursuivra. Dans le cas du mandarin Fum-Hoam en revanche, le couple corps-âme est plus déterminé par la composante physique : exposant le " dogme » (telle est la formule ici) de la métempsycose à son interlocutrice, le narrateur recourt à une imagerie métamorphique :

[...] notre âme est comme un caméléon, qui, suivant les différents corps où elle passe, y prend des impressions différentes, y est sujette à toutes les passions du corps qu'elle occupe [...]. J'ai paru dans toutes les parties du monde sous des formes très

23. J.-F. Perrin, «Petits traités de l'âme et du corps, les contes à métempsycose $\left(\mathrm{XVII}^{\mathrm{e}}-\mathrm{XVIII} \mathrm{I}^{\mathrm{e}}\right)$ », dans Le Conte merveilleux au XVIII siècle, une poétique expérimentale, Paris, Kimé, 2000, p. I23-I39.

24. Je me permets de renvoyer ici à mon étude : «Soi-même comme multitude : le cas du récit à métempsycose au XVIII ${ }^{e}$ siècle ", Dix-huitième siècle, nº 4I, 2009.

25. Béroalde de Verville, Le Voyage des princes fortunés (I610), Entreprise seconde, Dessein dixhuitième, Éditions passage du Nord/Ouest, 2005, p. 367. Raymonde Robert avait signalé cette filiation des Soirées Bretonnes au Voyage des princes fortunés. On lira avec profit la mise au point de Christelle Bahier-Porte, éditrice des Soirées Bretonnes dans l'ouvrage annoncé. 
opposées ; j'ai été par conséquent de toutes sortes de religions et de tout sexe, et j'ai, par un pouvoir singulier, conservé jusqu'à présent le souvenir des principaux faits qui sont arrivés sous mes yeux ou auxquels j'ai eu quelque part ${ }^{26}$.

Cette âme transmigrante est donc dotée par hypothèse d'une plasticité qui la rend spontanément accordée à son avatar. Mais à cet égard, l'intérêt d'un recueil comme les Aventures merveilleuses du mandarin Fum-Hoam tient sans doute beaucoup à l'éclairage sceptique dans lequel le récit cadre place cette croyance et son actualisation narrative ${ }^{27}$.

Des analyses récentes ${ }^{28}$ ont montré qu'une des caractéristiques du texte romanesque à cette époque, consiste en la reprise d'un discours qui crée une vraisemblance pour la mettre en question : cette poétique impliquant un travail sur la perception des formes narratives par le lecteur ; le "roman critique " se construit avec ce qu'il envisage de sa propre réception et des effets de celle-ci. Or à la vraisemblance près (pour le lecteur du recueil ${ }^{29}$ ), c'est un peu la situation qu'inscrit la fin de la première partie du récit cadre des Contes Chinois: le "programme » instauré par Fum-Hoam donnant la série autobiographique de ses avatars, admet en effet au départ le scepticisme et l'ironie de son auditrice (et donc a fortiori du lecteur) : « je sais (que votre majesté) est si prévenue contre les principes de notre religion, qu'elle regardera comme autant de fables des vérités qui en sont tout le fondement "; mais parallèlement, ce cadre suppose de leur part la suspension volontaire du jugement comme condition de possibilité de la narration ; en effet, exposant les principe de la transmigration des âmes et du ressouvenir des vies antérieures, il conclut : « c'est ce qu'il faut, madame, que vous ayez la bonté de me passer, quelque répugnance que vous ayez à le faire, si vous voulez entendre le récit d'histoires assez surprenantes, qui vous convaincront de la vérité de ce que je vous avance "; il s'agira donc d'écouter des fables en admettant qu'on puisse les entendre comme des vérités, ou de raconter des histoires véritables, tout en admettant que l'auditrice (et le lecteur) les considèrent comme des fictions. Nous sommes

26. Contes Chinois, ouvr. cité, t. I, p. 6o. Je modernise l'orthographe.

27. L'approche ironique de cette croyance est déjà celle du dialogue de Lucien intitulé Le Songe ou le Coq, où Pythagore incarné en coq, présente à Micylle la série de ses avatars. Ce scénario a été repris par Madeleine de Scudéry, dans une séquence de Clélie (IV, liv. II et III) où l'on persifle Damon en lui faisant évoquer ses supposés avatars.

28. J.-P. Sermain, Métafictions (I670-1730), Champion, 2002.

29. Comme l'écrit Montesquieu dans le projet d'avant-propos à l'Histoire véritable : "les Aventures du mandarin Fum-Hoam ont été regardées comme fabuleuses par tous les critiques. " (Euvres complètes de Montesquieu, 9, Oxford, Voltaire Foundation, 2006, p. I29-130) 
bien dans un récit "métafictionnel », dont voici le contrat, formulé par la princesse :

Je promets de vous écouter avec un plaisir extrême, et de ne point vous chicaner par des réflexions qui pourraient vous embarrasser ; j'attendrai, à vous proposer mes difficultés, que vous ayez achevé tout ce que vous avez à me dire, et que vous soyez parvenu à l'état du mandarin sous la figure duquel je vous vois à présent ${ }^{30}$.

Ce pacte sera pour l'essentiel respecté ; les interventions de Gulchenraz visant surtout à la relance des récits ; néanmoins, elle glissera par-ci par-là, quelques remarques elliptiques, signalant une contradiction entre l'histoire racontée et le "dogme » de la métempsycose ; par exemple après une histoire de revenants, ou après une histoire de voyage au pays des âmes. Et c'est ici au lecteur qu'est laissé le loisir de reconstituer l'objection.

Un autre aspect de cette dimension métafictionnelle apparaît dans les contes eux-mêmes, à l'occasion des séquences liées à la magie : outre quelques grandes scènes de sorcellerie ou de magie noire, on relève de nombreuses séquences où interviennent la cabale, l’alchimie, la magie naturelle, la science de Salomon, etc. Ce sont en général des récits initiatiques, le héros, avatar de Fum-Hoam où l'un de ceux dont il rapporte les discours, ayant été mis en contact avec ce savoir par un derviche, un fakir ou encore un "philosophe" au sens alchimique. Or dans ces séquences, on relève toujours des éléments de débat sur la crédibilité de cette sorte de savoir : de débat, car le récit finalement valide en général son efficacité ; pourtant l'intéressant reste bien l'installation d'un espace de doute, et cela sous deux aspects : soit un personnage tient un discours sceptique, soit le protagoniste narrateur demande une preuve, laquelle prend alors la forme d'une expérimentation sur l'animal. Par exemple, les pouvoirs d'un élixir de jouvence étant testés sur un vieil âne par le derviche Assirkan, la régénérescence de l'animal prend un certain temps, celui correspondant au récit des aventures du " philosophe "-alchimiste Abdal-Moal ; la surprise ici, est qu'au moment de se rendre auprès de l'âne, son interlocuteur se dit d'ores et déjà convaincu de ses pouvoirs par seule audition de son récit. Ainsi se trouve questionnée la pertinence du modèle empiriste (certes ici cousu de fil blanc) de rapport à la vérité : voici ce qu’on lit après constatation du rajeunissement de l'âne :

Hé bien, me dit alors Abdal-Moal, cela suffit-il pour satisfaire votre incrédulité ? Ah ! Seigneur, lui répondis-je, je ne suis que trop convaincu de la bonté de vos secrets ; je

30. Fin de l'« Histoire du sultan Tongluk », Contes Chinois, ibid., p. 6I. 
n'avais pas même besoin de cette épreuve pour y ajouter foi, le seul récit de vos aventures me suffisait, et elles m'ont paru aussi intéressantes que singulières ${ }^{31}$.

Résonne ici un intéressant contrepoint avec le scepticisme résolu de la sultane de Chine à l'égard de la vérité des aventures qu'on lui conte.

Il y aurait d'autres exemples du même ordre, dont on fera cependant l'économie en préférant montrer que la fin du recueil signale quasi explicitement sa dimension critique. Rappelons la façon dont se boucle le récit cadre : Gulchenraz Gundogdi et son père Malekalsalem ont été magiquement transportés dans leurs pays durant leur sommeil, où le vieux roi exilé sera rendu à son trône, ses adversaires ayant été éliminés. Durant la nuit, la jeune femme a fait un rêve où le vénérable mandarin Fum-Hoam est apparu sous les traits d'un jeune homme qui l'attire invinciblement; le lendemain, il s'avère que le mandarin était en réalité le frère de Gulchenraz disparu depuis sa naissance. Devenu au cours de ses aventures magicien et cabaliste musulman, il n'a jamais renié l'islam et c'est lui-même qui démontre in fine les inconséquences logiques et les impasses éthiques de la doctrine de la métempsycose ; mais c'est pour réciter, aussitôt après, tous les miracles attribués à Mahomet. Ici, un bref extrait :

Il punit les impies par les châtiments les plus terribles ; n'est-ce pas lui qui, pour châtier l'orgueil de Caïcaous, ordonna au moucheron de pénétrer jusqu'aux membranes de son cerveau, et de lui causer une douleur si insupportable, qu'il était obligé de se faire battre la tête avec un maillet : N'est-ce pas lui qui fit flotter sur la mer le corps de Feraoun avec sa cuirasse de fer $^{32}$ ?

Une note de l'auteur indique tout le bien qu'il faut penser de ce genre de haut fait dans la perspective chrétienne : "Suivant la même tradition, c'est Pharaon. Les Orientaux ont défiguré presque tout l'ancien testament, soit dans les noms, soit dans les faits »; et la note sur "Caïcaous » : "C'est, suivant l'histoire orientale, Nembrod ", va dans le même sens ; version contre version, aucune vérité prétendue n'est indépendante des textes. C'est toute la dimension ironiquement citationnelle du récit qui s’y dévoile du même coup ; on est très près ici de la poétique de Montfaucon de Villars dans le Comte de Gabalis : citer comme sérieusement des textes délirants, voire en développer les argumentaires de façon apparemment rationnelle, pour susciter chez le lecteur un réflexe ironique à leur égard.

3I. Contes Chinois, Xxxix soirée, "Suite des aventures du derviche Assirkan ", ouvr. cité, t. II, p. 205.

32. Contes Chinois, «Histoire d'Alroamat, et conclusion de l'histoire de Tongluk et de Gulchenraz Gundogdi », ibid., p. 332. 
Enfin, la cible de toute l'entreprise de récit étant en réalité le sultan de Chine, qui s'était engagé par amour à se convertir si Gulchenraz restait musulmane, Fum-Hoam-Alroamat révèle alors tout bonnement qu'il a forgé des contes à partir d'un corpus disponible, afin de le persuader :

Pour me prêter aux contes extravagants de vos mandarins de la loi, je vous ai raconté des histoires dans le goût de celles qu'ils récitent à tous moments, et dont quelquesunes sont arrivées; mais non pas à moi, qui n'ai jamais cessé d'être ce que je suis, que lorsque, par la vertu des paroles cabalistiques qui me sont connues, j'ai bien voulu paraître à vos yeux sous une autre figure ${ }^{33}$.

Ces contes étaient donc des forgeries, des élaborations secondes à partir de scénarios puisés dans une matière en circulation ; on ne définirait pas autrement le jeu littéraire de Gueullette : que ce soit avec la mode de la Chine en son temps, ou avec la matière narrative disponible sur les rayons de la Bibliothèque - et le type de lecture au second degré qu'il propose qu'on en ait.

Quelques mots dans ce fil, à propos de la reprise du thème d'Edipe dans l'" Histoire d'Houschenk et de Gulbaz » et l'" Histoire de la sultane Dugmé " insérée dans la précédente, car c'est encore un bon exemple de la façon critique dont Gueullette travaille avec l'ensemble de la bibliothèque ${ }^{34}$. En s'appuyant sur une trame qu'il emprunte à un article de d'Herbelot, il produit une version relativement intéressante de ce qu'on cherche à faire du sujet depuis Corneille : à savoir l'inclusion d'une intrigue amoureuse complète et une manipulation mieux dramatisée des signes équivoque du destin. Edipe est à la mode : celui de Voltaire date de 1718 (45 représentations successives), et en I726, ce sera celui d'Houdar de La Motte. Entre les deux, la version des Contes Chinois intervient en 1723 ; on retrouvera encore la thématique de l'inceste paternel dans Cleveland (I73I-I738). Nous savons en outre que c'est un thème que le conte merveilleux traite dans l'histoire de Peau d'Âne. On trouverait encore d'autres arrières textes jouant sur une méprise entre frères et sœurs de lait, par exemple chez $\mathrm{M}^{\mathrm{me}} \mathrm{d}^{\prime}$ Aulnoy, dans La Princesse Belle-Étoile. Dans l'" Histoire d'Houschenk et Gulbaze ${ }^{35}$ ", ce qu'apporte la paraphrase de l'article «Homai \& Khamani » tiré de la Bibliothèque orientale, c'est que Jocaste (la Dugmé du récit de Gueullette) a été violée dans son sommeil

33. Ibid., p. 325 .

34. Pour une approche critique moderne de ce récit, dans ses versions orientales originales, voir René Basset, Mille et Un Contes, récits et légendes arabes, t. II, au numéro IO2 : Les aventures de Dahab (J. Corti, 2005, p. I24-I27). La version de Gueullette est absente des références de Basset.

35. Contes Chinois, $\mathrm{vII}^{\mathrm{e}}$ soirée. 
à l'adolescence par un père qui a transféré sur elle son amour pour sa mère décédée ; on ne l'apprend que vers le milieu du récit. Ici, Edipe (Houschenk chez Gueullette) est un enfant trouvé par un teinturier qui l'élève avec sa fille Gulbaze, leur laissant ignorer l'origine inconnue du garçon. Gueullette apporte les éléments suivants : le nouvel CEdipe quitte sa famille adoptive par crainte d'un inceste avec celle qu'il croit sa sœur, et non par suite d'un oracle inquiétant ; comme CEdipe, il sauve le royaume, non par son adresse cependant mais par sa capacité guerrière, ce qui lui vaut la rencontre de la reine Dugmé. Deux rêves prémonitoires parallèles préparent les fiancés royaux à éviter l'inceste imminent, empêché in extremis grâce à la reconnaissance par la reine d'un signe distinctif sur la poitrine du jeune homme (cliché déjà repris dans le récit cadre des Contes Tartares), alors qu'ils se mettent au lit. Ensuite Houschenk vivra avec Gulbaze secrètement, tandis que la reine assume leurs enfants aux yeux du royaume. Elle-même meurt de mélancolie.

L'originalité de cette version est certainement d'abord l'insistance sur la souffrance psychique de cette femme violée par le père, qui est la seule véritable composante tragique du récit. Autre élément "moderne " au sens du XVIII ${ }^{\text {e }}$ siècle : l'insistance sur le double rôle préconscient de la " nature " : inciter (attrait)/séparer (répulsion) : c'est valable autant pour l'attrait des jeunes gens que pour ce qui se produit avec la reine. Troisième élément, pas d'arrière-plan mythologique : ici, Jocaste est fille de l'inceste et non descendante maudite du soleil. Tout se déroule dans un plan non surnaturel ; même les rêves sont interprétés par l'échauffement des imaginations et par les instincts de la nature. On note enfin, c'est sans doute un clin d'œil, que le nom du teinturier père adoptif d'Houschenk est Tocal, qui, selon la Bibliothèque orientale, signifie " le boiteux » - c'est-à-dire Edipe, comme on sait. On ne peut pas trouver une version plus démythologisée, plus désenchantée, de ce thème. Ici, Nature, forces politiques et hasard font tout : Gueullette semble avoir travaillé le scénario dans un sens agnostique et naturaliste ${ }^{36}$.

36. Pour être précis, cette histoire amplifie la matière de deux articles de la Bibliothèque orientale: les art. "Daráb » et « Homai \& Khamani » (eux-mêmes explicitement reliés par d'Herbelot, qui note à l'art. « Daráb » la similitude avec l'histoire de Moïse abandonné au Nil). Le premier présente l'histoire de l'enfant abandonné ; le second amène le thème de l'inceste subi par la sultane (l'inceste, évité in extremis, de celle-ci avec Houschenk, est sans doute de la plume de Gueullette). Pour mémoire, on retrouve le thème de l'inceste évité de justesse dans la parade (attribuée à Gueullette) intitulée L'Amant cochemard: Gille qui convoite Isabelle apprend à la fin de la pièce qu'elle est l'enfant qu'il a exposée jadis à La Salpêtrière. Commentaire : "J'eusse fait un inseq, queu bonheur ! mon bon Ange./ Me nouit l'aiguillet'. " (Théâtre des Boulevards, I, Paris, E. Rouveyre, I88I, p. 300) 
On l'a dit, une bonne part de la matière narrative de ce recueil et des suivants procède de la littérature documentaire, voire savante. Il arrive d'ailleurs parfois à l'auteur (plus rarement néanmoins dans les Contes Chinois que dans les Sultanes de Guzarate) d'indiquer en note le texte qu' il a utilisé. C'est le cas à propos de l'histoire du sauvage Kolao (XxIv soirée), histoire intégralement transcrite de la Nouvelle relation de la Gaspésie du père Chrestien Leclerq (Paris, I69I), sans modification significative du scénario de ce mythe chamanique, Gueullette se limitant à inventer le nom de Kolao et à effacer les commentaires théologiques ou apologétiques insérés par le missionnaire, dont le récit était destiné à illustrer les croyances des Gaspésiens sur l'immortalité de l'âme. Mais en général, Gueullette est extrêmement loin de donner toutes les sources de ses innombrables emprunts : dans le récit cadre par exemple, il plagie quasi littéralement un passage de l'article "Dab Schelim " tiré de la Bibliothèque orientale, pour construire l'histoire de la prise de pouvoir par Tongluk : chez d'Herbelot, Mahmoud le Gaznévide désire déléguer le pouvoir sur le royaume indien de Soumenat, qu'il a conquis, au plus proche descendant du dernier roi, lequel s'avère être l'ermite Dabschelim ; Gueullette modifie ce cadre en le sinisant et en l'infléchissant vers une situation de vacance du trône sans héritier direct, puis il reprend le scénario de la Bibliothèque orientale en le dialoguant. Cet emprunt lui sert à dramatiser efficacement la problématique de la succession royale qui est au centre des enjeux du récit cadre. Autre exemple d'emprunt quasi littéral : la séquence de l'histoire du prince Kader-Bilah ${ }^{37}$ où celui-ci (qui vient de vaincre le gardien d'une fameuse tour enchantée) rencontre Mohammed Mehdi, le XII ${ }^{e}$ prophète de la tradition chiite, lequel lui explique qui il est et lui montre une ville aux douze mille portiques qui est la cité paradisiaque de la fin des temps. Or toute cette description reprend textuellement l'article « Dunia » de la Bibliothèque orientale.

Dans une approche plus large de l'intertextualité des Contes Chinois, on peut considérer que Gueullette a paraphrasé ou colorié à l'orientale des scénarios épico-mythologiques archi-connus du merveilleux d'Occident - à moins que ce ne soit parfois l'inverse, conformément à la théorie de l'époque sur l'origine orientale des grands récits européens ${ }^{38}$. On a par

37. Contes Chinois, XLIII ${ }^{\mathrm{e}}$-XLVI ${ }^{\mathrm{e}}$ soirées.

38. On retrouve cela chez d'Herbelot : «L'on dit en Orient être transporté en Ginnistan, pour exprimer ce que nous dirions en français : être porté en féerie, comme parlent nos anciens romans. Car toutes les fictions et rêveries qui se trouvent dans ces ouvrages, sont prises, comme on l'a déjà remarqué, des romans et histoires fabuleuses des Orientaux. " (B.O., I697, p. 799). Pour avoir utilisé l'article " Mergian-Banou " dans l'" Histoire du prince Kader Bilah », Gueullette avait lu 
exemple une version musulmane du nouveau-né voyant aidant sa mère à échapper à une calomnie, que nous connaissons sous l'aspect de Merlin, mais dont Gueullette a trouvé la trame dans un article de la Bibliothèque orientale $^{39}$; quant à la XLIv ${ }^{\mathrm{e}}$ soirée du recueil, son début reprend la structure de l'histoire des rats de Hameln probablement puisée chez B. Bekker ${ }^{40}$, mais colorée à l'orientale par des éléments tirés de Chardin (cité en note) ou de la Bibliothèque orientale; mais si Gueullette hybride dans cette histoire des matériaux précis tirés de sources documentaires variées, il travaille aussi cette matière en la connotant des mythèmes du légendaire occidental comme le Minotaure ou le Morholt, et d'éléments reliés à la geste de Renaud et d'Armide; parallèlement, le légendaire musulman de la cité paradisiaque des élus est le cadre dans lequel s'achèvent les aventures du héros Kader-Bilah, délivreur des jeunes filles et de ses prédécesseurs malchanceux. Toute cette séquence est une lecture en partie double des deux mondes merveilleux et surnaturels occidentaux et orientaux.

Sur ce plan, le texte intègre également les genres équivoques ou les genres frontières comme le récit de rêve (beaucoup de rêves prémonitoires ou d'avertissement, généralement tragiques, mais pas exclusivement), lesquels genres jouent dans l'interzone du surnaturel et du merveilleux, avec par exemple l'hésitation caractéristique du fantastique dans le dernier récit : la tablette d'or contenant l'oracle du destin du héros se trouvant à la fois dans un livre ancien où il apprend son existence et au pied de la tour où il se rend (XLIV $-\mathrm{XLV}^{\mathrm{e}}$ soirées). L'ambivalence s'accentue avec les aventures d'Abdal-Moal et l'histoire d'Alroamat ${ }^{41}$, deux récits mettant en scène des vies de magiciens (dont l'un en mode " autobiographique »), qui sont aussi des récits d'initiation aux sciences secrètes (alchimie, cabale...) inscrites par leur praticien comme relevant plutôt d'une physique que d'une magie stricto sensu - on a déjà vu que la figure du " philosophe " se voile sous celle du magicien, la prudence imposant de mystifier le peuple, dans le droit fil de la tradition libertine «savante»:

que : «C'est du nom de cette fée que nos anciens romans ont formé celui de Morgane $[s i c]$ la Déconnuë.»

39. IV soirée, "Aventures de l’imam Abzenderoug ", ouvr . cité, t. I, p. IO7-II3. Voir dans la B.O. l'article " Gioraige ", consacré aux enfants ayant parlé dès le berceau.

40. B. Bekker, Le Monde enchanté, t. V, livre IV, chap. I9, \$2, Amsterdam, I694, p. 364-366; mais le XviII ${ }^{\mathrm{e}}$ siècle pouvait la lire in extenso (d'après Bekker) chez l'abbé Bordelon, dans L'Histoire des imaginations extravagantes de Monsieur Oufle, Amsterdam, I7IO, note du chap. 25.

4I. Contes Chinois, ouvr. cité, t. II, fin XXXIII ${ }^{e}$ soirée-XXXIX ${ }^{e}$ soirée et p. 297-322. 
Cachez toute la science que je vais vous communiquer sous un extérieur simple, modeste, et qui ne vous attire point l'envie des méchants. C'est la route que j'ai prise pour être parfaitement heureux : il est peu de malades qui viennent en ces lieux qui ne s'en retournent en santé ; croyez-vous que ce soient ces eaux qu'ils boivent ou dans lesquelles ils se baignent, qui fassent cette opération ? Non, mon cher ami, c'est moi seul qui apporte un souverain remède à leurs maux ; et pour vous le faire connaître, flairez seulement l'élixir qui est dans cette petite fiole : Je la débouchai, poursuivit Alroamat, je la portai à mes narines, et à peine eus-je respiré la vapeur qui en sortait, que je sentis un dérangement extraordinaire dans toutes les parties de mon corps ; et de courbé que j'étais, je me levai aussi droit que je pouvais l'être ; j'en pourrais faire autant à tous ceux qui viennent à Sargultzar, continua Koda-Bendé, mais ces prodiges m’attireraient bientôt la haine des médecins; je guéris mes malades peu à peu, et je leur fais croire qu'ils en ont l'obligation aux eaux de cette fontaine ${ }^{42}$.

Littérairement, ces séquences forment aussi une composante déterminante de la dynamique du recueil, car ce sont en général de longues histoires fertiles en rebondissements, aboutissant aussi à l'initiation de l'auditeur qui devient lui-même un adepte et un pratiquant initié à la "philosophie naturelle ", ce qui relance la machine narrative (et notamment le récit cadre, puisque Alroamat s'initie seul à la cabale avant de devenir l'élève d'un magicien puissant pour acquérir finalement les pouvoirs qui le feront passer pour Fum-Hoam). Du point de vue du rendement diégétique, il s'agit là d'une forme très proche dans ses possibles narratifs, du conte à métempsycose, car le secret de la jouvence éternelle et le pouvoir de faire de l'or autorisent bien des expériences humaines ou animalo-humaines dans l'espace et dans le temps.

Aussi bigarrées que l'oiseau fabuleux des Chinois dont Gueullette a trouvé le nom chez le père Kircher ${ }^{43}$, mais aussi peu chinoises que possible en dépit de leur titre ${ }^{44}$, les Aventures merveilleuses du mandarin Fum-Hoam

42. Ibid., p. 319-320. Ce personnage de Philosophe-alchimiste ironique à l'égard des croyances et superstitions, et capable de les manipuler à l'occasion, a certains des traits qu'on attache à la figure du libertin "érudit " du XvII ${ }^{\mathrm{e}}$ siècle, aussi audacieux en pensée que méprisant à l'égard des ignorants et prudent dans la vie sociale.

43. "On trouve de certains oiseaux dans la Chine qu'on ne voit pas dans les autres royaumes, du nombre desquels je mets en premier lieu cet oiseau royal qu'il appellent Fum Hoam [...], oiseau d'une admirable beauté [...]. Le mâle s'appelle Fam et la femelle Hoam. [...] Les mandarins du roi même ont accoutumé de porter la représentation de ces oiseaux en or sur leurs habits pour marquer quelque chose, et pour leur servir de symbole. » (Athanase Kircher, La Chine illustrée, Amsterdam, I670, p. 263, col. a)

44. En dehors du titre et du récit cadre, ce recueil n'a rien de chinois : sa géographie est indopersane pour l'essentiel, et sa culture "orientale " de référence est celle de l'islam vue à travers la Bibliothèque orientale et Chardin ou Tavernier. L'image de la Chine que présente le récit cadre est fantaisiste, qu'il s'agisse du système du mandarinat ou des questions religieuses, alors qu'on dispose depuis le XVII siècle d'une documentation précise. Sur ce point, voir V. Pinot : La Chine et 
tentent certes de capter une nouvelle fois le goût du public pour le conte oriental $^{45}$; littérairement cependant, ce recueil n'échappe pas à ce qui se passe dans ce chantier en plein bouleversement qu' est la prose narrative du premier tiers du XVIII ${ }^{\mathrm{e}}$ siècle. Cette époque bouillonne aussi d'idées nouvelles qui vont devenir celles des Lumières, mais que les œuvres saisissent où reflètent, pour un temps encore, à l'état plutôt germinatif ou conjectural ; mais si le "roman critique " se construit avec ce qu'il envisage de sa propre réception et des effets de celle-ci, et s'il le fait le plus lisiblement chez Gueullette comme affichage didactique dans l'annotation de bas de page, il le fait aussi plus subtilement - quoique systématiquement - en mode sceptique ou discrètement ironique, comme dispositif soigneusement agencé d'anecdotes et de récits tirés des dépôts de savoirs et de croyances que recèle la Bibliothèque; enfin, que la mystification y soit de règle à l'égard des esprits superstitieux, chez les adeptes de la philosophie naturelle, signale bien l'économie critique d'une narration du savoir qui inclut toute une palette de mises en récit possibles - de fictions plus ou moins mystifiantes autrement dit -, selon la qualité des destinataires.

\section{Savoir et croyance : une encyclopédie fabuleuse}

Cette poétique critique se développe et se raffine dans le recueil des Sultanes de Guzarate; on verra comment Gueullette y développe son style très équivoque de l'" insert " didactique, en bas de page ou directement dans le récit, puis on reprendra la question du "métafictionnel », ici explicitement reliée à la polyphonie énonciative d'un récit cadre où les narrateurs/auditeurs ne s'assurent d'un savoir qu'à le rapporter à une culture

la formation de l'esprit philosophique en France (1640-1740), Paris, 1932, réimpr. Slatkine, Genève, I971, et Dix-huitième siècle, $\mathrm{n}^{\circ} 8$ (numéro spécial sur les Jésuites), Garnier, 1976. Pour un état de la bibliographie récente, voir Bruno Neveu, "La Chine en Europe ", Dix-huitième siècle, n 28, PUF, I996, p. I35-I40.

45. En I723, l'intitulé " chinois " est encore presque neuf dans la prose narrative comme au théâtre. En croisant les répertoires de Marie-Louise Dufrenoy (L'Orient romanesque en France, 1704-I789, t. II, Montréal, 1947) et de Frédérique Balliot (Chinoiseries littéraires, la Chine et la littérature d'imagination en France de 1704 à 1789, Thèse, Lyon 3, 1996), on obtient un roman de Du Bail : Le Fameux Chinois (I642), deux œuvres de $\mathrm{M}^{\mathrm{me}}$ de Villedieu : Axiamire ou le Roman chinois (1675), Nouvelles et galanteries chinoises (1712), et deux histoires dans les recueils de contes antérieurs : "Histoire des amours de Camaralzaman [...] et de Badoure Princesse de la Chine " dans Les Mille et Une Nuits (1705-1706), "Histoire du prince Calaf et de la Princesse de Chine " dans Les Mille et Un Jours (1710-1712). Au théâtre de la Foire, on joue Arlequin invisible chez le Roi de la Chine (Lesage, représentation en 1713), et Arlequin, Barbet, pagode et médecin, pièce chinoise de deux actes en monologue (Lesage et d'Orneval, représentation en I723). 
partagée des mondes de la fiction. On réfléchira enfin à la portée de l'importation et du commentaire d'extraits d'un document d'époque sur la religion musulmane, pour esquisser une idée de la façon dont ce recueil fait insister ce qu'on pourrait appeler la question de la vérité comme effet de texte.

Gueullette insiste, on l'a vu, dans l'avant dire de ce recueil, sur l'innovation qu'y représente le développement des notes documentaires par rapport aux usages en cours dans le genre. À cet égard, ces Contes Mogols se caractérisent par une explicitation jamais poussée à ce point dans les précédents recueils - ceux de Gueullette compris -, des sources documentaires utilisées. Il pousse la précision jusqu'à donner l'édition, le tome, le folio de ses références, exactement comme on le pratique jusqu'à nos jours dans l'édition savante ; on peut ainsi lire en contrepoint des récits, une sorte de chronique ethno-documentaire appelant le lecteur à se reporter à la source indiquée quand une note a pu susciter sa curiosité. Il parle de "notes géographiques et historiques un peu plus amples que l'on n'a coutume de le faire en pareil cas ", et met en exergue "l'autorité de la Bibliothèque orientale " pour les notes concernant " l'Histoire fabuleuse ", insistant, pour finir, sur la quantité de temps et de lecture que lui a demandé un tel travail d'annotation ${ }^{46}$. Ce qu'il y laisse apparaître est cependant seulement la partie émergée de l'iceberg. En effet, les extraits qu'il donne en bas de page ne livrent qu'une petite partie du matériel utilisé pour l'écriture des Sultanes : une part essentielle en est en effet directement intégrée au récit, notamment pour la matière anecdotique (voir en particulier l'" Histoire d'Aboul-Assam ${ }^{47}$ ", nourrie sur ce plan par Chardin, Lucas, Le Bruyn, Vincent, d'Herbelot, etc. $\left.{ }^{48}\right)$. Quant à la matière des notes elles-mêmes, elle est intéressante à plusieurs titres. Au premier plan, la documentation tirée principalement de d'Herbelot et des voyageurs en Orient (ceux que tout le monde connait, Chardin surtout, mais aussi Grelot, Leblanc, Olearius, Van Sleb), concernant les mœurs liées à l'intrigue : nombre d'épouses autorisé par le Coran, danses érotiques à la cour de Perse, certaines coutumes idolâtres, rituels de pèlerinage à La Mecque, notion du Kauli, mythologie persane, signification des noms propres, etc. En outre, on rencontre également des références aux coutumes des Indiens d'Amérique de Nord, tirées des Mours des sauvages

46. Cf. ma note 17.

47. Les Sultanes de Guzarate, $\mathrm{XVI}^{\mathrm{e}}$-XXIII ${ }^{\mathrm{e}}$ soirées.

48. Pour la démonstration sur pièces, je renvoie à l'annotation et à la notice de mon édition des Sultanes, au vol. 9 de la « B.G.F. » chez Champion, à paraître en 2010. 
américains, du père Lafitau (1724) dont est recopié en note un long passage en XXIV $^{\text {e }}$ soirée ${ }^{49}$, sur les différents aspects du rite de la " couvade " au Canada et en Europe, envisagés selon l'hypothèse comparatiste chère au fameux jésuite. Cependant, Gueullette est loin de dévoiler toute sa documentation : par exemple, ici, sa lecture de l'Histoire de l'Amérique septentrionale, de La Potherie (I722), dont des paragraphes entiers sont insérés dans le récit même pour l'épisode du séjour du prince Cazan-Can sur l'île Ramak (xxiv ${ }^{e}$ soirée également) : cette greffe concernant le différé de la consommation du mariage dans les usages indiens, permet à Gueullette de tirer son héros d'une situation délicate parmi les anthropophages d'une mer peu attendue en tel contexte référentiel, puisqu'il s'agit de la mer d'Oman ${ }^{50}$.

En effet, si d'un point de vue géographique, les Contes Mogols couvrent un vaste territoire incluant l'Inde de l'ouest, la péninsule arabique, la Perse et le Kazakhstan, Gueullette n'a aucune gêne, comme c'était déjà le cas dans les Contes Chinois avec les "Aventure du sauvage Kolao ", à intégrer dans son texte et ses notes des données ou des anecdotes concernant d'autres parties du monde. Une légende phrygienne sur les rats trouvée dans la Géographie de Noblot, cautionne ainsi un épisode de la geste d'Abderaïm en Carizme (LXXI ${ }^{\mathrm{e}}$ soirée) ${ }^{\text {sI }}$. C'est aussi un épisode de la Vie de Christophe Colomb qui sert, en fin de xxvi ${ }^{\mathrm{e}}$ soirée, à faire échapper Cazan-Can et son pilote au bûcher des sauvages, par prédiction

49. Contes Mogols, t. II, ouvr. cité, p. 77-78.

50. Version de Gueullette : Le héros pour sauver sa vie est contraint de demander en mariage la fille du chef, mais il redoute la nuit de noce avec un tel " monstre "; le pilote du navire naufragé le console en lui présentant les longs délais qu'imposent les mœurs maritales de la peuplade : "Suivant donc le conseil de cet homme [...], j’allai le lendemain à l'entrée de la nuit dans la cabane d'Agariata ; je la tirai trois fois par le nez pour l'éveiller : comme c'est une cérémonie essentielle, je n'eus garde d'y manquer. Cette belle fille ne me dit aucune parole, elle se contenta de me regarder d'un air riant, à la lueur d'une petite lampe que je tenais à la main ; et le tout s'étant passé avec beaucoup de circonspection et encore plus de bienséance, je me retirai très content de la modestie de ma jeune maîtresse, et je fus obligé, pendant plus de deux mois, de renouveler toutes les nuits pareille cérémonie. » (Ibid., p. 84-85). Version de La Potherie (non référencée par Gueullette) : «La manière dont un amant fait paraître à sa maitresse l'estime qu'il a pour elle lorsqu'il la regarde en vue du mariage, est extrêmement bizarre. Les petits entretiens familiers ne se font que la nuit, en présence de quelques amis ; l'amant entre dans la cabane de la fille qui n'est fermée ordinairement que d'une peau volante [...], et s'approchant de sa maitresse, il lui tire le nez par trois fois pour l'éveiller ; ce qui est une formalité essentielle : tout se passe avec bienséance, sans que la fille lui dise aucune parole. Ces sortes de témoignage d'amitié durent près de deux mois avec beaucoup de circonspection de part et d'autre. " (Claude-Charles Le Roy Bacqueville de la Potherie, Histoire de l'Amérique septentrionale, ch. 3, Paris, I722, rééd. O. Delavault, D. Dubois, I. Zéo, Monaco, éd. du Rocher, 1997, p. 236)

5I. Ouvr. cité, t. III, p. 223-224. 
d'une éclipse ${ }^{52}$ (Hergé retrouvera l'idée !). Plus près de la géographie de sa fiction, il utilise en LXIve soirée, pour un épisode de "roman alchimique "se déroulant à Schiraz ${ }^{33}$, une séquence tirée des Voyages [...] faits en Moscovie, Tartarie et Perse d'Olearius, d'ailleurs déjà pillée par Steele pour le Spectator. Toujours au titre des collages " interculturels » dont il a le secret, on peut noter qu'un des rares apports de Gueullette a son plagiat complet de La Calprenède ${ }^{54}$ dans l' " Histoire de Canzadé ", consiste dans la mention en note de bas de page d'un extrait de Chardin sur les origines fabuleuses de l'inceste selon la mythologie persane : la notion de "Kauly» est donc censée venir éclairer un épisode du Cléopâtre - habillage oriental obligess. Autre exemple, la grande fée Mergian-Banou que les Contes Chinois avaient présentée en redoutable ginne dans le cadre d'une réécriture orientalisante de la légende du flûteur de Hameln tirée de Bekker, devient dans les Contes Mogols (LXVIII ${ }^{\mathrm{e}}$ soirée) la fée bénéfique par excellence, dans un récit dont la matrice est l'histoire de Fortunio chez Straparole. Dans les Contes Chinois, Gueullette avait fabriqué le grand prêche final d'Alroamat comme un centon de prélèvements coraniques dans la Bibliothèque orientale, associés avec certains éléments de la critique lucrétienne des idées pythagoriciennes sur la métempsycose ${ }^{56}$. Il ne l'avait pas explicité en note. Dans les Contes Mogols en revanche, il livre sa source pour tous les passages concernant la description du paradis musulman : ce sont plusieurs pages consécutives de La religion ou théologie des Turcs d'Echialle Mufti (I7I4) ${ }^{57}$ qui passent directement dans le récit du voyage en songe d'Abderaïm au paradis (LXXVIII ${ }^{\mathrm{e}}$-LXXIX ${ }^{\mathrm{e}}$ soirées). Le

52. Ibid., t. II, p. I06-II3. Source non référencée par Gueullette.

53. Ibid., t. III, p. I4I-I45. Le scénario est celui popularisé par Balzac dans L'élixir de longue vie. La critique balzacienne (Cf. notamment Bruce Tolley, "The Source of Balzac's Elixir de longue vie ", Revue de littérature comparée, $\mathrm{n}^{\circ}$ 37, 1963, p. 94) renvoie à la version de Steele dans le Spectator, $\mathrm{n}^{\circ}$ 426, 9 juillet I7I2 (elle-même d'ailleurs tirée des Voyages d'Olearius); mais elle ignore l' "Histoire d'Aboul-Assam ", également sans doute directement paraphrasée d'Olearius. (J'utilise l'édition Amsterdam, 1727, t. II, p. 667-668.)

54. Il s'agit l'" Histoire d'Olimpie » dans Cléopâtre de La Calprenède, plagié à la phrase près de la $\mathrm{VI}^{\mathrm{e}}$ à la XIII ${ }^{\mathrm{e}}$ soirée. Le second tiroir emprunté à ce romancier pour l'" Histoire de Zelm-Alzaman $[\ldots]$ et de Zenderoud [...]" (fin XXIX -XLI $^{\mathrm{e}}$ soirées) est l'" Histoire d'Alcamène roi des Scythes et de la reine Menalippe ", prise aux livres II-IV de la vIII ${ }^{\mathrm{e}}$ partie du Cléopâtre.

55. En viI ${ }^{\mathrm{e}}$ soirée.

56. Contes Chinois, ouvr. cité, t. II, p. 325-328. La démonstration d'Alroamat paraphrase pour l'essentiel les arguments développés par Lucrèce au livre III du De Rerum natura, vers 670, sv.

57. Il n'y a guère de documentation sur ce texte étrange (qui fut mis à l'index en 1709) et son auteur. Albert Monod y soupçonne une mystification (De Pascal à Chateaubriand, les défenseurs français du Christianisme de 1670 à 1802 (1916), rééd. Burt Franklin, New York, 1971, p. 280). 
recueil de contes apparaît ainsi comme une machine textuelle à mettre en corrélation divers secteurs de la Bibliothèque, sans que le point de vue qui organise l'ensemble soit assignable ailleurs que dans la poétique de la disposition et de l'agencement du tout ; dans l'ensemble, cette poétique consiste quasi exclusivement en un art du montage et de la greffe, à toutes les échelles du travail : l'invention, Gueullette la prend chez autrui, et la diction est chez lui une affaire de prélèvements et de collage, de paraphrase et de condensation.

Le second point pouvant caractériser l'apport spécifique du recueil des Sultanes touche à un approfondissement (ou une sophistication) de la dimension métafictionnelle déjà observée dans les Contes Chinois. Ici, ce sont les rapports de détermination réciproque de la croyance (et des savoirs dont elle prétend s'autoriser) au discours de la fiction. Pour le saisir, il faut brièvement situer le recueil dans l'histoire des formules européennes d'encadrement narratif : à cet égard, on peut dire que le récit cadre des Sultanes de Guzarate fait revenir dans la matrice importée des Mille et Une Nuits certains caractères des formules issues aussi bien de la nouvelle à devisants multiples que de la tradition romanesque issue de L'Astrée. Par rapport à la structure du cadre narratif dans les recueils orientaux antérieurs, l'un des aspects originaux du récit cadre des Sultanes est que l'auditoire du palais va s'agrandir de presque tous les survenants qui, une fois racontées leurs aventures, deviennent hôtes et auditeurs. Alors que chez Galland et Pétis, l'auditoire actif est en pratique réduit à une ou deux personnes, on retrouvera ici un "personnel » de plus en plus comparable sur le plan quantitatif, à celui des recueils à la Boccace et surtout à ceux de la "Romancie». Avant les Sultanes d'ailleurs, les recueils orientaux de Gueullette jusqu'aux Contes Chinois, reprennent grosso modo le modèle fondamental issu des Nuits : un roi ou une princesse sont distraits de leurs soucis ou d'une obsession par une conteur ; et si comme chez Pétis de la Croix, les récits du mandarin des Contes Chinois, sont censés provoquer une conversion de la princesse (ici à l'islam, là à l'amour de l'autre sexe), ils n'ont aucun effet sur ce plan en réalité, cette conversion ne résultant pas d'un dialogue effectif mais étant dans les deux cas liée à des procédures (dé)mystificatrices indépendantes et gérées dans le récit cadre.

Les conteurs de la lignée de Scheherazade racontent de mémoire des histoires dont ils ne sont pas les protagonistes ; ce n'est plus le cas du mandarin des Contes Chinois, qui délivre un récit autobiographique de ses aventures transmigratrices; c'est cependant son point de vue qui domine, même si parfois deux de ses avatars présentent des versions différentes 
des mêmes faits ${ }^{58}$. Dans les Contes Mogols en revanche, les polarités s'inversent radicalement : il n'y a plus ni auditeur ni narrateur uniques et les structures des deux sphères aussi bien que leur interaction sont profondément différentes. Le pôle des auditeurs est ainsi hiérarchisé : une écoute invisible qui subordonne le plaisir à la volonté d'espionnage, c'est celle d'Oguz, un sultan qui se fait passer pour mort un certain temps (selon d'ailleurs un scénario bien repéré sur le plan politique dans les relations ${ }^{59}$ ) afin de vérifier l'amour de ses cinq épouses ; personne, sauf l'iman Cothrob, magicien ordonnateur de tous les prestiges, ne sait que cette oreille-là enregistre tout ce qui se dit dans le salon des sultanes. L'auditoire visible est constitué des cinq sultanes, de Cothrob et des deux enfants du sultan ; c'est un auditoire mystifié (Cothrob mis à part) dont les réactions sont conditionnées par sa croyance à la mort réelle du sultan; mais c'est aussi un auditoire mystifiant, encouragé aussi bien par les survenants que par Cothrob, à se faire passer pour surnaturel et omniscient. Il est acquis en effet dès le premier récit, que ces soi-disant peris savent de science surnaturelle tout ce qu'on va leur raconter, mais que ce qui leur importe est l'intention et la manière, car elles sont réputées avoir la capacité d'aider efficacement leurs hôtes s'ils s'en montrent dignes à leurs yeux. Autrement dit, cet auditoire est supposé évaluer la qualité morale des protagonistes selon leur style narratif. Ce pouvoir se vérifie d'ailleurs assez vite à l'usage, par le biais de l'omnipotence de Cothrob qui joue efficacement son rôle d'adjuvant surnaturel (en liaison avec d'autres actants de son niveau) visà-vis des attentes exprimées ou secrètes des protagonistes-narrateurs. Le troisième niveau de la sphère auditrice est constitué par les voyageurs-narrateurs eux-mêmes, dont certains restent au palais tant que leur situation n'est pas réglée et qui, en tant que tels, peuvent être amenés à reprendre la parole lorsque tel ou tel personnage qui leur importe sort de derrière la fameuse " portière " par laquelle on fait entrer au salon du palais les nouveaux hôtes enlevés au caravansérail de Cambaye. C'est l'instance la plus complètement mystifiée, évidemment, même si comme on le verra, c'est aussi de ce niveau que sortent les réflexions sur la vérité de la nature surnaturelle du lieu et des maîtres.

58. Comparer dans les Contes Chinois, la "Suite [...] de l'Histoire de Magmu» (Xviri ${ }^{\mathrm{e}}$ soirée) et la "Suite de l'Histoire du vizir Houssan ben San » (xxIII ${ }^{e}$ soirée) $:$ le crime est vu par le témoin puis par le criminel.

59. Ainsi que l'a noté Alain Grosrichard dans son étude de ce corpus : «Le despote est toujours comme mort, et c'est à cette condition que son nom s'élève à la plus haute efficacité symbolique. " (Structure du sérail, ouvr. cité, p. 98-99). Cf. aussi Montesquieu : "Les hommes sont tels dans ce pays qu'ils n'ont besoin que d'un nom qui les gouverne. " (L'Esprit des Lois, Livre V, ch. I5) 
Si l'ampleur de la palette "sociale » du recueil de contes orientaux est un des éléments de leur attrait depuis Galland, il semble indéniable que l'originalité sur ce plan des Contes Mogols est la part accordée aux narrateurs liés au monde des spectacles et des fictions : Karabag, qui ouvre le cycle des soirées, dirige une troupe de musiciens ; Canzadé, pour être princesse, n'en chante pas moins dans sa troupe au moment où le lecteur fait connaissance avec elle ; Aboul-Assam, l'aveugle de Chitor, a été bouffon du roi à une époque de sa vie ; Massoud, fils de Soffar, joue le rôle de Megnoun devant les sultanes, séduisant la sultane Goul-Saba puis racontant sa propre histoire hautement picaresque ; il cède ensuite la parole à Mouïad, un autre acteur de sa troupe, donné pour spécialiste du genre merveilleux-véridique, en bon émule d'un père surnommé "Khedab" (le menteur) pour son obstination à faire passer pour vrai un récit de sa vie apparemment fabuleux. Cette présence importante des artistes dans le personnel des narrateurs a une répercussion importante, voire décisive sur la nature des débats qui se déroulent au sein du récit cadre. On ne débattait guère, finalement, dans celui des Contes Chinois, avant l'épilogue du recueil et le grand prêche d'Alroamat, sur les vertus de l'Islam ni sur le caractère éventuellement fabuleux du Coran. Si l'on avait en revanche quelques débats sur la poétique des récits et de leurs enchaînements au fil des Contes Tartares, et quelques pages fameuses sur la comparaison du merveilleux féerique et du merveilleux oriental dans la lettre préface des Aventures d'Abdalla, on trouvait fort peu à cet égard dans les Mille et Un Jours et dans l'Histoire des Sultanes de Perse. Il faut attendre la trilogie d'Hamilton en I730, pour voir s'ouvrir dans le conte oriental un débat entre l'auditoire et le conteur sur l'art de raconter. Or deux ans après le conte des Facardins, et deux ans avant l'irruption décisive de Crébillon dans le champ du conte merveilleux, la réflexion sur le rapport entre fiction et vérité, entre surnaturel et réel, est devenue un fil rouge de l'ensemble du récit cadre des Sultanes de Guzarate. Il n'est sans doute pas sans signification que ce soit la directrice d'une troupe de musiciens qui le lance à la fin de la première partie du récit cadre, en posant face au pessimisme d'un autre membre de sa troupe, que le palais dans lequel ils se trouvent est conforme à ce qu'enseignent les « anciens romans » :

Ma chère fille [reprit-elle] je crois avoir pénétré le mystère de cette aventure : tous les anciens romans turcs et persans dont j'ai fait autrefois la lecture, sont remplis d'événements bien plus merveilleux; si ce qui se passe en ce moment n'est pas l'effet d'un songe, il faut que nous ayons été transportés pendant cette nuit dans le Ginnistan, et tous les objets que nous voyons ici, doivent être de cette belle espèce de créatures 
que l'on appelle peris ou perises, qui ne sont produites que pour faire du bien aux hommes et pour soulager les malheureux ${ }^{60}$.

L'admission du surnaturel comme surréel est une donnée immédiate de l'univers de référence des personnages des Mille et Une Nuits ou des romans de chevalerie ; mais l'instauration d'un débat sur les critères d'accréditation du surréel comme plan de réalité admissible aux yeux des personnages du récit cadre, semble une caractéristique du recueil de Gueullette. Le point de vue attribué à Karabag revient d'ailleurs en d'autres segments du cadre : par exemple, à la fin des "Aventures d'Aboul-Assam " (XXıII soirée), il est dit que les princes Cothbedin et Cazan-Can informés par Canzadé du caractère surnaturel du palais et de ses maîtres,

comme ils avaient lu dans les anciens romans plusieurs aventures à peu près pareilles, ils crurent possible que les mêmes puissances qui avaient conduit la princesse en ces lieux les y eussent également transportés pour y terminer leurs peines ${ }^{61}$.

Cependant, il existe aussi dans le récit cadre des instances pour mettre en doute cette croyance et ses attendus; le plus incisif est Aboul-Assam l'aveugle de Chitor, qui éclate de rire à l'idée de se trouver en Ginnistan, arguant précisément de la culture qu'il possède de la littérature merveilleuse : "Ah, ah, dit-il, cela est nouveau ; et ce palais est-il situé dans la ville de Gabkar ou dans celle d'Anbaradad ? Car tu vois que je sais un peu la carte de ce pays " $\left(\mathrm{XV}^{\mathrm{e}} \text { soirée }\right)^{62}$. Il ne perd d'ailleurs rien pour attendre puisque sa guérison miraculeuse (quoique en réalité obtenue par magie naturelle) le mystifiera si bien qu' elle vaudra à l'auditoire une vibrante apologie de Mahomet, du coup placé aux yeux du lecteur (ou de l'auteur) dans le même plan que les héros de romans ou de légendes (ibid.). Un autre endroit où le doute est installé sur la crédibilité de l'accréditation du surréel par les vieux romans, est la présentation que donne de la légende de Meignoun et Leïleh, la vieille qui mène la troupe de danseurs accueillis en LXII ${ }^{\mathrm{e}}$ soirée :

Nous ne pouvons pas nous imaginer qu'il y ait des amants si sots et des filles si réservées, nous n'avons pas conservé dans notre pièce les caractères tels que les romans nous les dépeignent ${ }^{63}$.

6o. Contes Mogols, ouvr. cité, t. I, p. II8.

6r. Ibid., t. II, p. 67. Le sel du commentaire est que leurs aventures à tous trois (à peu près un quart de l'ensemble du recueil) sont effectivement issues d'un ancien roman de France, Cléopâtre de La Calprenède!

62. Ibid., t. I, p. 300. Une note à cet endroit renvoie à la B. O. à propos du caractère "fabuleux " de ces villes.

63. Ibid., t. III, p. I24. 
Toutes ces indications signalent bien dans le récit cadre de ce recueil, un plan de réflexion où l'agencement ambigu du discours romanesque oblige le lecteur à s'interroger sur le degré d'adhésion des personnages aux croyances qu'ils paraissent véhiculer. Mais la nouveauté décisive en est ici la narrativisation et la mise en discours dans les voix de la sphère conteuse. Du coup, le débat sur l'accréditation de la croyance par les anciennes fictions devient un des enjeux explicites des dialogues développés dans le récit cadre. Ce débat intervient principalement au niveau des visiteursnarrateurs, soumis par leur auditoire et les précédents arrivés à s'imaginer en présence de peris, et amenés à constater qu'effectivement ils trouvent en ces lieux l'aboutissement de leurs quêtes, par l'intervention d'auxiliaires dont les capacités excèdent ceux de la simple humanité. C'est ce que pensent les deux princes liés à Canzadé, une fois leurs difficultés résolues : c'est ainsi que Cazan-Can doit reconnaître en Cothrob le vieillard qui, dans un rêve antérieur, lui a surnaturellement retiré du cœur la graine maléfique (elle-même prélevée dans le Coran via la Bibliothèque orientale) qui le rendait amoureux passionné de sa sœur ; ainsi est-il d'autant plus " porté à $[. .$.$] ajouter foi » à l'idée qu'ils se trouvent en Ginnistan, que «ce qu'il$ venait de dire à l'iman marquait que ce n'était pas la première fois qu'il avait vu ce grand homme " (fin de la XXIII ${ }^{\mathrm{e}}$ soirée) ${ }^{64}$.

"Je ne sais si je songe ou si je veille ", c'est ainsi souvent que s'expriment d'abord les nouveaux arrivants au palais des sultanes, à peine sortis des torpeurs provoquées par le soporifique au "bueng " qui leur a été administré pour les transporter sans encombre depuis le caravansérail de Cambaye. C'est généralement pour admettre peu de temps après qu'ils sont bien éveillés en pays de Ginnistan, autant dire Romancie, mais une Romancie qui passe à leurs yeux pour réelle ; aussi réelle que le sont pour Don Quichotte et Sancho mystifiés, un fameux château et une non moins fameuse île. Avec le cinquième et dernier cycle du recueil dont le récit sera d'abord lancé par une second danseur de la troupe de Massoud, c'est ce mélange instable de mensonge et de vérité qui vient sur le devant de la scène ; Massoud présente en effet son compagnon comme un expert en récits merveilleux : récits dont il est d'ailleurs seulement le relais puisqu'il les tient de son propre père, lequel en atteste la vérité (fin de la XXVI soirée). Massoud est celui qui dans le recueil est obligé par hypothèse à dire la vérité sur sa propre histoire, sans quoi il subirait de graves représailles (fin de la LXII ${ }^{\mathrm{e}}$ soirée) ; néanmoins beaucoup d'aspects de son histoire touchent à l'extraordinaire voire au merveilleux ; par exemple, lors

64. Ibid., t. II, p. 67. 
de son éducation par l'alchimiste qui lui a servi de père adoptif, il a manipulé un élixir d'immortalité, certes si maladroitement qu'il l'a gâché (pas pour toute le monde car Balzac pourrait l'avoir exhumé du Cabinet des fées pour sa propre nouvelle) ${ }^{65}$. Mais c'est sans doute à ses performances don juanesques qu'il pense, lorsqu'il annonce à l'auditoire " des histoires qui tiennent encore plus du merveilleux " pour introduire celles de son ami Mouïad, lequel " assure sur la foi de son père " que "quelque incroyables qu'elles paraissent [...] il n'y a pas un mot d'imaginé ${ }^{66} »$. Et son relais de confirmer dès le commencement de son récit :

Je suis fils d'un homme qui avait servi dans les troupes du sultan de Candahar avec assez de distinction : il s'appelait Abderaïm, et il racontait des choses si singulières qui lui étaient arrivées, que la plupart de ceux qui l'écoutaient n'y ajoutant aucune foi, lui avaient donné le surnom de Kedhab.

Une note en bas de page précise ici que ce mot signifie "le Menteur ${ }^{67}$ ». Et le fils du Menteur poursuit sans ciller son introduction, en annonçant qu'il va commencer par l'histoire de sa naissance : "Comme j'étais presque toujours présent à ces récits, voici ce que je lui ai ouï dire, entre autres, de l'événement qui avait donné lieu à ma naissance. "Si les idées freudiennes sur le roman familial ont quelque valeur, c'est le cas de dire que voilà un fils qui aurait des raisons de penser être fils de roi plutôt que fils d'Abderaïm-le-Menteur. Mais on est entré dans le domaine hautement paradoxal du menteur-qui-dit-la-vérité dont la Romancie philosophique nous dit que le lignage est crétois, et dont toute la tradition du conte atteste qu'elle se confond avec son propre territoire : qui dit conteur dit menteur : "Je ne suis point payé pour vous dire la vérité» dit une formule classique du contage oral, et c'est une grande conteuse française contemporaine qui nous rappelle que "si tu veux être cru alors rends la vérité incroyable $"{ }^{68}$. Mouïad y revient un peu plus loin en évoquant l'opinion des voisins de son père à son sujet : celui-ci raconte en effet (c'est le récit rapporté par Mouïad aux LXVIII ${ }^{\mathrm{e}}$-LXXIV ${ }^{\mathrm{e}}$ soirées) avoir épousé la princesse de Carizme, Zarat-Alriadh, obtenue en combattant sous la forme d'un lion avec l'aide de la fée Mergian-Banou, en récompense d'un service rendu à celle-ci ; puis avoir été enlevé au fond de la mer par la ginne Scheïtan-Couli au cours d'une partie de pêche, peu de temps après son mariage ; puis avoir été délivré par la princesse selon des moyens

65. Cf. ma note 53 .

66. Contes Mogols, LXvi ${ }^{\mathrm{e}}$ soirée, ibid., t. III, p. I68.

67. Ibid., fin de LXVI ${ }^{\mathrm{e}}$ soirée, p. I69.

68. Muriel Bloch, La Sagesse de la conteuse, Paris, L'œil neuf éditions, 2008, p. 29. 
magiques, puis l'avoir à nouveau perdue par suite d'un enlèvement maritime ; après quoi il est revenu dans son pays avec son fils, craignant de revoir son royal beau-père sans avoir auparavant retrouvé son épouse. Et Mouïad ajoute alors :

Comme toutes les espérances de mon père, malgré sa situation présente, n'étaient pas entièrement évanouies, il se plaisait à se rappeler souvent l'heureux temps auquel il avait joui d'une fortune si brillante ; il avait des voisins avec lesquels il s'entretenait souvent de ses aventures; et quoiqu'il les racontât toujours de la même manière sans jamais se contredire, ils les trouvaient si incroyables, qu'ils lui donnaient souvent en riant, comme je vous l'ai déjà dit, le surnom de Kedhab dont Abderaïm ne se fâchait pas, convenant lui-même que si un autre lui faisait de pareils récits, il aurait toutes les peines du monde à y ajouter foi ${ }^{69}$.

À partir de la LXXvi $^{\mathrm{e}}$ soirée, la suite de la narration des aventures d'Abderaïm est assumée par le héros lui-même devant l'auditoire habituel du palais. Le premier fil en est la quête de l'épouse disparue, mais l'intrigue se double d'un second fil ayant trait à nouveau à la dimension du merveilleux, mais un merveilleux d'une autre sorte sinon d'une autre essence que celui de la féerie, puisqu'il s'agit du surnaturel religieux musulman. Abderaïm se qualifie en effet sur le terrain religieux en étant à l'initiative, sur inspiration du Prophète suggère-t-il, de l'éradication de l'idolâtrie dans la région d'Agra en Perse. Ayant surpris des "bramins " du dieu Ram préparant cyniquement la défloration d'une jeune vierge consacrée au Dieu, il perturbe la cérémonie et ameute la population, qui se convertit en masse à la Vraie Religion non sans avoir mis au bûcher les prêtres de l'idolâtrie. Cette performance missionnaire lui vaut d'être introduit en songe au paradis des vrais croyants, qu'il visite en compagnie d'un ange, durant les sept années qu'il lui reste à attendre, selon son destin, pour retrouver définitivement la princesse son épouse.

Pour évoquer le plus concrètement possible la topographie et les habitants du paradis musulman, Gueullette n'a pas procédé comme à la fin des Contes Chinois où il avait utilisé à cet effet ce qu'il avait trouvé dans la Bibliothèque orientale. Il a cette fois utilisé une autre source documentaire : cette Religion ou Théologie des Turcs par Echialle Mufti, à laquelle j'ai fait allusion plus haut. Il avait utilisé les chapitres 48 et 49 du tome 2 pour le récit cadre des Sultanes, dans une longue note sur les houris, et dans une explication sur le " Poul serrha ". Il récidive à l'autre extrémité du recueil, en reprenant cette fois sur plusieurs pages en LXXVIII $^{e}$ et LXXIX ${ }^{e}$ soirées, l'histoire de la visite de Gabriel au paradis selon le Prophète. Après

69. LXXIV e soirée, ouvr. cité, t. III, p. 253-254. 
quoi, le héros est transporté à la mosquée de Tangut où son talent lui vaut d'être remarqué par le sultan qui le recrute pour prêcher une femme de son sérail dont il s'avérera qu'elle n'est autre que Zarat-Alriadh son épouse.

Chez Chardin et d'Herbelot, tout développement documentaire sur le contenu religieux de l'islam est toujours assorti d'une clausule méprisante sur l'imposture du prophète, le caractère " fabuleux " des récits fondant la révélation musulmane, leurs contradictions éclatantes, et leurs honteux plagiats de la Bible. C'est exactement l'esprit dans lequel Gueullette présente sa source, que ce soit à l'entrée du recueil où dans les dernières soirées : « tant d'extravagances et de puérilités avec des détails aussi ridicules, sont rapportés dans le second volume de la religion des Turcs, par Echialle Mufti dans les chapitres 48 et 49 depuis le fol. 96 jusqu'au Io9 "; "Quoique les Mahometans prétendent que leur Prophète ait été garanti du péché originel et de la concupiscence, ils ne peuvent se dispenser d'avouer qu'il a eu vingt-et-une femmes contre la loi qui n'en permet que quatre "; "il est difficile de concevoir comment des gens sensés peuvent ajouter foi à de pareilles puérilités, dont le détail est encore infiniment plus circonstancié dans ce livre "; " voilà ce qui s'appelle un véritable pays de Cocagne. Voyez Echialle Mufti, partie 2, fol. $108^{70}$ ". Mais comme pour toute la littérature libertine dénonçant la superstition religieuse, la question est de savoir quelle est exactement la cible de Gueullette, sous "l'orientale allégorie » dont parle Hamilton à propos de Galland ${ }^{71}$. On n'aura garde d'oublier ici en effet, que le narrateur autobiographique du voyage au paradis en songe raconté auX LXXVIII ${ }^{\mathrm{e}}$-LXXIX ${ }^{\mathrm{e}}$ soirées, n'est autre que celui que tous ceux qui le connaissent surnomment « le Menteur »; le menteur, c'est le conteur - la bibliothèque vivante des récits merveilleux et extraordinaires, qui donnent l'illusion de la vérité mais qui sont en réalité des fictions. Gueullette sait très bien qu'il touche ici à l'argumentaire de Galland préfaçant le premier volume de sa traduction des Contes Arabes. Ceux-ci, annonçait-il, sont à la fois plus extraordinaires et merveilleux qu'aucun conte connu en Europe, et sont en même temps plus utiles pour connaître les réalités de l'Orient que les récits des voyageurs eux-mêmes. Quant une fois le Menteur a dit la vérité - et il l'a dite puisque Cothrob le réunit à sa femme et à son fils en présence des sultanes -, ce qu'il raconte de son voyage au paradis relève-t-il de la vérité propre au surnaturel religieux ou du mensonge propre aux affabulations bien connues des « mahometans " ?

70. Ibid., respectivement, t. I, p. 109 et p. 74 ; t. III, p. 290 et 294.

7I. A. Hamilton, Les Quatre Facardins, Paris, Champion, «B.G.F.", vol. I6, Hamilton et autres conteurs, 2008 , p. 538 . 
Dans le champ du conte oriental depuis les Nuits, il n'y a pas de difficulté : les plans du réel et du surnaturel préadamite, féérique, islamique ne sont jamais étanches les uns par rapport aux autres. Dans les Contes Arabes, un être humain ordinaire peut tuer un génie à son insu pour avoir jeté un noyau de datte dans le désert; un pêcheur peut retourner en sa faveur la magie du sceau de Salomon, un génie peut rencontrer dans les airs une fée plus puissante que lui parce qu'elle a fait allégeance au dit Salomon, etc. Dans les Contes Mogols, Abderaïm peut découvrir la plus puissante des fées dans la vieille qu'il a trouvée au fond d'un sac accordé comme butin de guerre par son général ; le prince Cothbedin peut comprendre sur l'île de Darem que les épreuves qui l'y attendent sont cohérentes avec ce qu'il avait jusque-là pris pour des fables; quant au palais de Cambaye, sa tangible surnaturalité est attestée pour ses hôtes par les romans et les légendes qu'ils connaissent. En contexte chrétien en revanche, ce genre de recodage continue à poser problème depuis la querelle du merveilleux chrétien et du merveilleux païen au XVII ${ }^{\mathrm{e}}$ siècle $^{72}$; et un auteur comme $M^{\text {me }}$ Leprince de Beaumont éprouvera encore la nécessité de faire distinguer aux petites élèves de son institutrice, la différence entre le surnaturel vrai de la Révélation et le surnaturel fabuleux des contes de fées ${ }^{73}$. L'ironie des notes de bas de page de l'auteur des Contes Mogols à l'égard du surnaturel musulman, s'autorise implicitement de l'autorité du christianisme ; mais en même temps, le lecteur européen est invité à identifier dans chaque histoire les vieux romans et les contes de France, les nouvelles italiennes, et bien sûr de fameuses visites littéraires au paradis, notamment la rencontre d'Astolphe et de saint Jean sur la Lune au chant Xxxiv de L'Arioste, pour ne citer que cet exemple. Gueullette n'est ni Montesquieu ni Voltaire, c'est certain, mais il participe du courant général de mise en question plus ou moins joueuse, plus ou moins sceptique, de la « fable » chrétienne sous couvert d'ironie à l'égard de la fable musulmane. On comprendrait mal sinon, qu'il consacre tant de soins à colorer d'Orient soigneusement documenté une matière narrative pour l'essentiel européenne, en encadrant en quelque sorte son recueil par de larges extraits, en bas de pages au début puis dans l'épilogue de la diégèse même, d'un ouvrage de vulgarisation de la religion musulmane.

72. Voir Aurélia Gaillard, Fables, mythes, contes. L'esthétique de la fable et du fabuleux (1660-1724), ch. 2, Paris, Champion, 1996.

73. Leprince de Beaumont, Le Magasin des enfants, t. I, III ${ }^{e}$ dialogue et VII ${ }^{e}$ dialogue, Lyon, I804, p. 36-37 et I24-I25. 
C'est ici le lieu enfin, de remarquer que le sage Cothrob est présenté lors de son apparition dans le récit cadre, comme "le neveu du célèbre Alroamat ", célébrité de roman bien sûr, comme le suggère la note de Gueullette à cet endroit renvoyant le lecteur à " [ses] contes chinois, imprimés à Paris chez Saugrain, Prault et Morin en 1723 ", l'invitant froidement à s'y reporter " au folio 297 du second volume ", et lui expliquant pour finir, pince sans rire, que "ce fameux cabaliste " a réussi à convertir la Chine à l'Islam, et que son neveu ayant succédé à son père sur le trône de Chine, l'a abandonné pour se consacrer à la vie contemplative en Inde ${ }^{74}$. Mais Gueullette n'a pas manqué au même endroit, de signaler que le nom de ce mystique oriental signifie "lutin, esprit follet " - ce que confirme la Bibliothèque orientale; on ne peut alors que sourire quand la fin du récit cadre désigne encore emphatiquement le "sage Cothrob» comme descendant du "grand Alroamat75 ». Dans le long épilogue des Contes Chinois, le prêche de ce dernier contre les contradictions du "dogme " de la métempsycose n'est qu'un centon de prélèvements de tout ce que la Bibliothèque orientale peut compter de fabuleux dans l'islam, doublé d'un argumentaire contre la métempsycose puisé chez ce grand croyant qu' est Lucrèce $^{76}$ ! Contradictions pour contradictions, Gueullette ne traite pas la matière religieuse et morale autrement que la matière narrative qu'il collecte sur tous les rayons de la bibliothèque, dans les romans, les recueils de nouvelles, les traités savants et moins savants, les relations, etc. Tout lui est bon pourvu qu'il soit ou puisse devenir matière de récit ou de discours dans ses recueils. Et s'il sous-titre finalement ses Sultanes " ou les songes des hommes éveillés ", c'est bien sûr parce que nul n'ignore en son temps rococo, que " la vie est un songe " au sens de Calderon (qu'il a d'ailleurs adapté pour son ami Riccoboni) ; cependant, sur ce fond de scène, le débat feutré mais insistant ouvert dans l'ensemble du récit cadre des Sultanes, touchant la légitimation de la croyance (et au fond de la religion) par l'autorité des fictions reçues, constitue certainement une étape importante vers la façon dont les dialogues caustiques de Crébillon sur l'art de conter comme art de penser, réinventeront la question du pouvoir des fables.

Avec les recueils de contes de Gueullette, on se trouve en présence d'une étonnant dispositif rhétorique où tout l'art du récit consiste à disposer

74. Contes Mogols, ibid., t. I, p. 8I.

75. Ibid., t. III, p. 345.

76. Cf. ma note 56 . Je renvoie pour le détail de la démonstration à mon édition du recueil. 
ingénieusement une matière pour l'essentiel empruntée, selon une poétique très travaillée de la variété, des contrastes réglés et des enchâssements bien bouclés. Très peu d'invention à proprement parler, peut-être aucune, tant il semble que le plaisir d'écrire se soit ici intégralement confondu avec celui de monter des plagiats de différents niveaux et d'échelles variables : art de la greffe et de l'hybridation, art du collage et de l'agencement méticuleux, des alternances de registres et de climats, de l'entrelacs des fils narratifs, des interactions entre dispositifs encadrants et " soirées » encadrées. L'art de Gueullette est à cet égard un bon témoignage de l'effet de la machinerie fictionnelle complexe introduite par Galland, dans le champ d'une esthétique du récit en plein bouleversement dont les tendances profondes, chez les grands romanciers "modernes", ne vont pas avant tout - il faut le marquer nettement - à l'achèvement ni au bouclage sans reste de l'entreprise narrative : le roman français du XVIII ${ }^{\mathrm{e}}$ siècle, parfois le meilleur, est en effet souvent inachevé, ouvert, "in progress "; et d'ailleurs, quant Hamilton, dans le champ du conte, a voulu s'en prendre aux Mille et Une Nuits, il a évidemment cassé la cohérence des emboîtements ou les a qualifiés ironiquement dans ses récits cadres déconstruits. Là n'est pas le choix de Gueullette dont la poétique n'est pas d'invention mais de composition, et dont les recueils constituent des sortes de micro bibliothèques des récits où l'on peut trouver l'imitation orientalisée de tout ce qui a cours en la matière sur l'étal des libraires du XviII ${ }^{\mathrm{e}}$ siècle ; avec y compris des tendances comme le récit utopique, la robinsonnade, ou ce qu'on appellera plus tard le récit fantastique (puisque les traités de démonologie sont aussi d'excellents gisements de matière narrative). Gueullette serait au fond plus un " passeur » qu'un auteur bien qu'il se revendique tout de même tel en donnant le recueil de ses œuvres dans son testament; quoi qu'il en soit, il recueille et transmet : son intérêt dans l'histoire de la prose narrative de son temps serait d'avoir produit une machinerie à tirer du narrable de tous les secteurs de la Bibliothèque, et notoirement de ces branches de discours réputées prétendre au savoir et à la vérité - et, qu'il l'ait voulu ou non, d'avoir offert à ses lecteurs matière à comparer, à croiser, à hybrider discours de savoir et matière de fiction. Comme une textualité généralisée peut-être, dont ces recueils cassent les ordonnances, mélangent les pages et recomposent les textes, en questionnant les savoirs par les fictions, les discours par les récits, les croyances par les fables, et vice versa, car prélever et monter, c'est aussi révéler la textualité fictionnelle des Écritures prétendument révélées ou des connaissances censément assurées. Entre les contes d'Hamilton et ceux de Crébillon, les contes orientaux de Gueullette attirent l'attention de leurs lecteurs sur les 
procédures d'accréditation de la croyance, ils inscrivent mœurs, savoirs et religions comme reliées à l'économie générale des fictions ; c'est ainsi moins dans leur propos explicite ou affiché que dans leur agencement et leur polyphonie qu'ils constituent, à leur façon, des fictions critiques. 